\title{
ALTERNATIVE NATIONAL MILITARY STRATEGIES \\ FOR THE UNITED STATES
}

Conrad C. Crane

Reporting Author

December 2000 